\title{
Stricture prevention after extended circumferential endoscopic mucosal resection by injecting autologous keratinocytes in the sheep esophagus
}

\author{
Barbara F. Zuercher • Mercy George • \\ Anette Escher · Elsa Piotet · Christos Ikonomidis • \\ Snezana Blant Andrejevic $\cdot$ Philippe Monnier
}

Received: 28 March 2012 / Accepted: 3 July 2012/Published online: 6 September 2012

(C) Springer Science+Business Media, LLC 2012

\begin{abstract}
Background During the past decades, endoscopic mucosal resection (EMR) has been developed to treat early intramucosal esophageal cancers and dysplastic Barrett's esophagus. The primary drawback of this method is severe postsurgical esophageal stricture formation. The purpose of this preclinical study was to assess strategies for prevention of this major complication by injecting autologous keratinocytes in the EMR mucosal defect in the sheep model.

Methods Circumferential, 6-cm-long EMRs were performed in the esophagus of nine sheep. Autologous keratinocytes were harvested 2 weeks before EMR and cultured. Circumferential resection consisted of two opposite hemicircumferential mucosectomies allowing a widespread resection of $24 \mathrm{~cm}^{2}$. Immediately after EMR, autologous keratinocytes were endoscopically injected in the mucosal defect. Animals were sacrificed after 6 months.

Results Circumferential EMRs were successfully performed in all animals. There were no intra- or postoperative complications. None of the animals developed strictures. All animals were sacrificed at 6 months as planned. Histological
\end{abstract}

B. F. Zuercher - M. George - A. Escher - E. Piotet .

C. Ikonomidis $\cdot$ P. Monnier

Head and Neck Surgery, Department of Oto-Rhino-

Laryngology, University Hospital (CHUV), Lausanne,

Switzerland

B. F. Zuercher ( $\square)$

Head and Neck Surgery, Department of Oto-Rhino-

Laryngology, Inselspital, University of Bern, 3010

Bern, Switzerland

e-mail: barbarahauler@hotmail.com

S. B. Andrejevic

Institute of Pathology, University Hospital (CHUV), Lausanne,

Switzerland examinations showed fibrotic changes in $10 \%$ (range 0-25\%) of the circumferential muscularis propria interna layer and $7.2 \%$ (range 0-25\%) in the muscularis propria externa layer at the midportion of the EMR. No circumferential transmural fibrosis was identified.

Conclusions Prevention of stricture formation after extensive (6-cm long) circumferential EMR of the sheep esophagus can be achieved by injecting autologous keratinocytes into the wound of the resected mucosal segment.

Keywords Stricture prevention - Autologous keratinocytes $\cdot$ Endoscopic mucosal resection

With the routine use of panendoscopy as well as abrasive sponge cytology during the follow-up of patients with head and neck cancers, a significant number of esophageal cancers are detected at an early stage as second primaries [1]. Until the past decade, the only curative surgical option was esophagectomy (fraught with perioperative mortality and morbidity) or brachytherapy with cumbersome multiple treatments. With the development of endoscopic mucosal resection (EMR), a new therapeutic alternative became available [2-5]. The procedure is minimally invasive and can be offered to patients who are not eligible for esophagectomy as a curative treatment. Early intramucosal esophageal cancers up to stage T1a that do not invade the submucosa and show no vessel infiltration, as well as dysplastic Barrett's esophagus or superficial adenocarcinomas arising in Barrett's esophagus, have a very low risk of lymph node metastasis [4-7].

When used for small lesions $(\leq 3 \mathrm{~cm}$ in diameter and circumferential tumor size $<60 \%$ ), EMR is a relatively straightforward procedure with few complications [4]. However, for more extensive lesions, postoperative stenosis 
is a major concern that occurs more frequently when the mucosal defect is larger than $30 \mathrm{~mm}$ in length or with a circumferential extension of more than $60 \%[4,5,7]$. The risk of stenosis can be as high as $62 \%$, depending on the extent of the circumferential resection [2]. The resulting dysphagia is unacceptable for initially asymptomatic patients. Treatment of these strictures consists of repeat bougienage or balloon dilatation with suboptimal improvement of symptoms and potential risks of tears and perforation $[2,8]$. With multicentric foci of early squamous cell carcinomas or early adenocarcinomas arising in dysplastic Barrett's esophagus, the curative treatment requires complete removal of the cancerous and dysplastic mucosae. This implies extensive circumferential resection in most of the cases, but effective methods that prevent stricture formation currently do not exist.

The application of intramucosal prophylactic steroids and currently available stents cannot prevent stricture formation [9]. Several studies have reported the use of 5-fluorouracil [10] to prevent stricture formation, along with autologous urinary bladder extracellular matrix [11], fatty tissue-derived stromal cells [12], and buccal keratinocytes [13] injected into the resected area. Most of these studies have tried to promote the process of wound healing by covering the mucosal defect to recreate a barrier function and to avoid long exposure of the submucosa to food and refluxate. In full-thickness skin wounds secondary to burns, cultured skin is commonly used to prevent stricture formation and contraction of the regenerated skin $[14,15]$. We hypothesized that cutaneous keratinocytes obtained by skin-tissue engineering technologies might promote early reepithelialization when injected into the mucosal defect resulting from EMR to avoid inflammation, ulceration, and cicatricial stenosis of the esophageal wall, chronically exposed to saliva, food, and refluxate. The purpose of this preclinical study was to investigate the preventive effect of injecting autologous cutaneous keratinocytes in the wound of circumferential mucosectomies to prevent stricture formation in the sheep model.

\section{Materials and methods}

\section{Study design}

This study was approved by the Animal Care and Use Committee of our institution. Nine sheep weighing 44-61 (mean, 50.3) $\mathrm{kg}$ were used. The sheep model was chosen because its esophagus is very similar to that of the human, especially the histological structure and thickness of the wall [16, 17]. All experimental procedures were performed under general anesthesia. The sheep were premedicated with Xylazine (Rompun $2 \%$, Bayer) and intubated endotracheally after anesthesia was induced by Disoprivan (Propofol $1 \%$, Fresenius). Anesthesia was maintained by inhalation of Isoflurane (Isoflurane ad us vet, Minrad). At the end of the follow-up period, the animals were euthanized with an overdose of pentobarbital sodium injected intravenously.

Isolation of keratinocytes and initiation of culture

Autologous keratinocytes were harvested from the anesthetized sheep 2 weeks before EMR. The inguinal region was shaved, washed with antibacterial soap, disinfected with Betadine, and rinsed with sterile saline solution. A $5-\times 10-\mathrm{cm}$, split skin graft, 2- to 4-mm-thick was obtained with a dermatome. The samples were washed with phosphate-buffered saline solution (PBS, Bichsel AG), cut with a scalpel into small pieces of epidermis, placed into flasks of medium, and attached on the surface with one drop of Tissucol 1.0 (Lyophilisate, Baxter) per 5-ml medium. The medium (DMEM/Dulbecco modified Eagle medium, Gibco BRL) contained $2 \%$ fetal bovine serum (FBS, Gibco BRL), essential amino acids, $2 \mathrm{mMol}$ of glutamine, $100 \mathrm{U} / \mathrm{ml}$ of penicillin, $100 \mu \mathrm{g} / \mathrm{ml}$ of streptomycin, and $2.5 \mathrm{U} / \mathrm{ml}$ Fungizone. After 1 week, the medium was replaced by one containing only $0.25 \mathrm{U} / \mathrm{ml}$ of Fungizone. Cultures were incubated at $37{ }^{\circ} \mathrm{C}$ in a humidified $5 \%$ $\mathrm{CO}_{2}$ atmosphere. Normal cell growth and lack of infection were controlled regularly during the 2 -week period. In case of contamination or insufficient proliferation, harvesting of new keratinocytes was repeated.

Cultures were trypsinated with $0.05 \%$ trypsin and $0.02 \%$ ethylene-diamine-tetra-acetic acid before being injected into the wound of the EMR. The cells were counted with a hemocytometer, centrifugated, and resuspended in $20 \mathrm{ml}$ of PBS. Finally, $0.3 \mathrm{ml}$ of Tissucol 0.1 per $20 \mathrm{ml}$ of PBS was added.

Endoscopic mucosal resection procedure and injection of keratinocytes

The EMR device and technique have been described previously by Radu et al. [2, 3, 18, 19]. The EMR was performed with a modified esophagoscope (Karl Storz GmbH \& Co, Tuttlingen, Germany), which is similar to a standard rigid esophagoscope with a length of $49 \mathrm{~cm}$. It is composed of an outer tube with a hemicircumferential window of 6-cm length situated on its distal end. An inner tube that seals the window and thus avoids mucosal tears during positioning was mounted on a $0^{\circ}$ telescope used for visual control. Once the lesion was targeted, the inner tube was removed and the resectoscope was introduced into the outer tube (Fig. 1). The resectoscope consists of a disposable cartridge with a perforated transparent window containing a $30^{\circ}$ telescope that permits precise targeting of the 


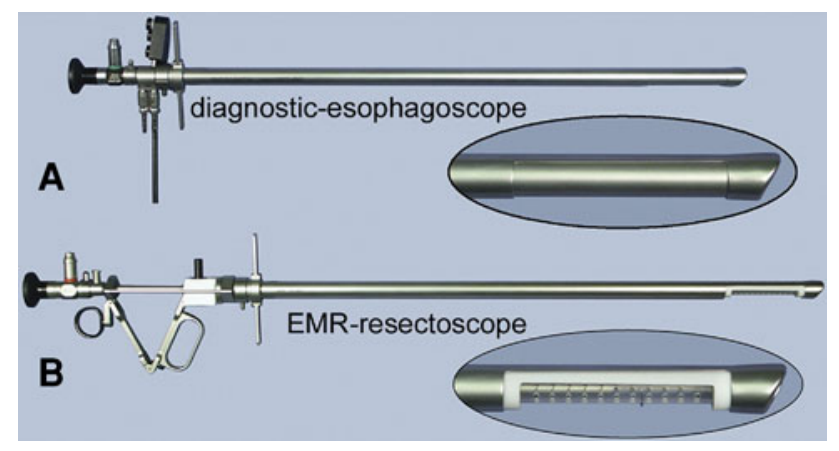

Fig. 1 A The diagnostic esophagoscope (Karl Storz GmbH \& Co, Tuttlingen, Germany). B The modified esophagoscope (Karl Storz $\mathrm{GmbH} \& \mathrm{Co}$, Tuttlingen, Germany) with inner tube and $0^{\circ}$ telescope for correct positioning in the esophagus and angulated $30^{\circ}$ scope and mobile wire loop

lesion through the window. After positioning, negative pressure was activated in the system, which sucked the mucosa against the transparent window. The system was set at a predetermined submucosal depth that was maintained at $1.1 \pm 0.1 \mathrm{~mm}$. A thin $(0.3 \mathrm{~mm}$ in diameter $)$ diathermy wire loop allowed the resection of a $6-\times 2-\mathrm{cm}$ $\left(12 \mathrm{~cm}^{2}\right.$ ) mucosal segment in one single piece (Fig. 2).

For circumferential resections, two hemicircumferential EMRs were performed on opposite sides. Thin residual mucosal strips that may have persisted after the two opposite resections were easily removed with a standard biopsy forceps. The EMR was preceded by circumferential submucosal injection of $50-100 \mathrm{ml}$ of adrenaline saline solution $(0.01 \%: 1 \mathrm{mg}$ of adrenaline in $100 \mathrm{ml}$ of saline solution) over a $10-\mathrm{cm}$ length to lift the submucosal layer from the muscularis propria layer, thus obtaining a regular cut in the submucosa without any bleeding. The EMR was performed in the esophagus of all sheep at a distance of $25-35 \mathrm{~cm}$ from the incisor teeth, over a length of $6 \mathrm{~cm}$.

Following the EMR, a mean of $17.8 \times 10^{6}$ (range, $13.2-25 \times 10^{6}$ ) autologous keratinocytes suspended in $20 \mathrm{ml}$ of PBS solution were endoscopically injected in the wound of the mucosal defect by using a standard rigid esophagoscope (Karl Storz GmbH \& Co, Tuttlingen,

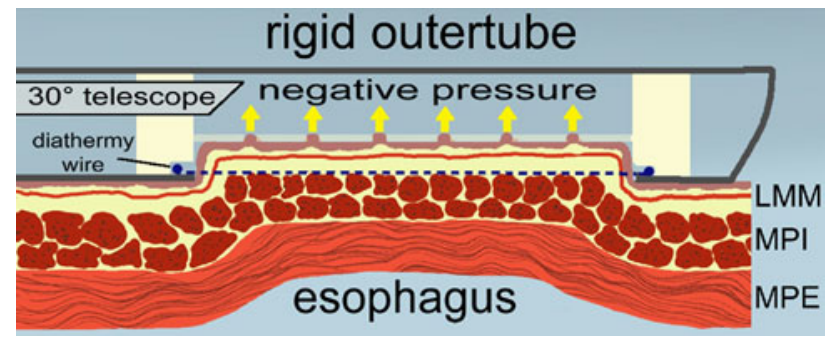

Fig. 2 Technique of EMR: the sucked mucosa is sectioned with the mobile wire loop under visual control. $L M M$ lamina muscularis mucosae, MPI muscularis propria interna, MPE muscularis propria externa
Germany). Through a Williams' injection needle, $0.5-1 \mathrm{ml}$ of autologous keratinocytes were injected circumferentially at equal intervals into the exposed submucosal tissue at 20-30 different sites.

\section{Postoperative care}

Following the procedure, the sheep were given daily Marbofloxacinum (Marbocyl $10 \%$, Vetoquinol) intramuscularly as a prophylactic antibiotic treatment and $1 \mathrm{~g}$ of paracetamol (Pro-Dafalgan, UPSA) for analgesia for 7 and 5 days, respectively. After EMR, the animals were fed a special diet without straw or hay to minimize the risk of injury and food impaction. A normal diet was resumed after 8 weeks, when reepithelialization was thought to be complete. During this period, food intake and weight were initially controlled daily and then at 3-day intervals. When there was insufficient food intake or weight loss of more than $10 \%$, an endoscopy was immediately performed. Otherwise, routine endoscopy was not planned during the follow-up period until the final control at 6 months to avoid any additional injury to the esophageal wall. The sheep were placed in environmental control facilities accredited by the CHUV Hospital.

\section{Histological analysis}

After euthanizing the sheep, the cervical esophagus was harvested for macroscopic examination and histological control. The rigid esophagoscope was introduced up to the hilt, its tip reaching beyond the resected area. A longitudinal incision was made in the neck; the trachea was identified and resected cranially up to the level of the larynx. This provided easy access to the esophagus. Normal inflation through the esophagus was used to determine any esophageal stenosis. Strong overinflation associated with occlusion of the distal esophagus with a Kocher clamp was used to identify any zone of restricted dilatability at the midpoint of the former circumferential EMR. This zone of relative stricture was then marked by placing a stitch on the adventitia of the esophagus. After sampling of the esophagus, the specimen was stretched to its original length and fixed in $5 \%$ buffered formalin. At the level of the previously marked midpoint of the EMR, two transverse circumferential sections at a distance of $5 \mathrm{~mm}$ were taken to analyze the deepest zones of fibrosis. In the remainder of the specimen, anterior and posterior longitudinal sections proximal and distal to the midpoint of the EMR were taken to measure the length and depth of fibrosis over the entire length of resection, identified histologically by a loss of the muscularis mucosae. The sections were embedded in paraffin and stained with hematoxylin-eosin and Masson's trichrome stains. The length of the reepithelialized area, 
characterized by a lack of lamina muscularis mucosae was measured. The depth and extent of scarring through the submucosa and muscularis propria were evaluated in the circumferential and longitudinal sections.

\section{Results}

\section{Clinical findings}

Circumferential resection over a 6-cm length was achieved in all animals $(n=9)$. There were no procedural complications, such as handling problems, massive bleeding, perforation, or death. Dysphagia with moderate food intake was observed during the first 6 (range, 4-7) days after EMR in five of the nine sheep. During the observation period, no weight loss was observed. An average weight gain of body mass of $11.5 \%$ (range, 2-16.3\%) and $29.3 \%$ (range, 14.9-63.8\%) was recorded at 1 and 6 months, respectively (Fig. 3).

\section{Endoscopic findings}

Control endoscopy was part of the protocol only at the end of the follow-up period unless there were unusual circumstances. In one sheep, an interim control endoscopy was needed at the ninth postoperative week because of diminished food intake without any weight loss after reintroduction of a normal diet with hay and straw. Endoscopy did not reveal any stenosis or impacted food, and the postoperative course was uneventful. At 6 months, all sheep underwent a final control endoscopy. On normal inflation, no stricture was observed, and the luminal surface of the resected area was flat, completely reepithelialized, and sometimes had a brownish discoloration.

Macroscopic and microscopic analysis

At the end of the procedure, the mean length of denuded esophagus was 7.3 (range, 6-9) cm, even though an EMR of $6 \mathrm{~cm}$ in length was performed. The mean length of the resected mucosa, including the LMM, was 44.2 (range,

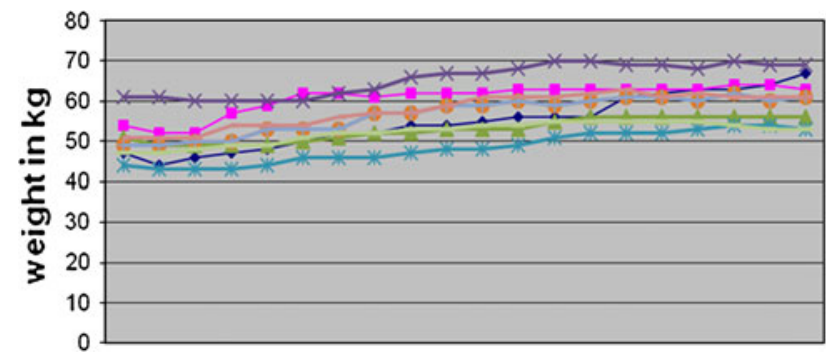

Fig. 3 Weight curve of the sheep during the first 10 weeks (measured twice weekly)

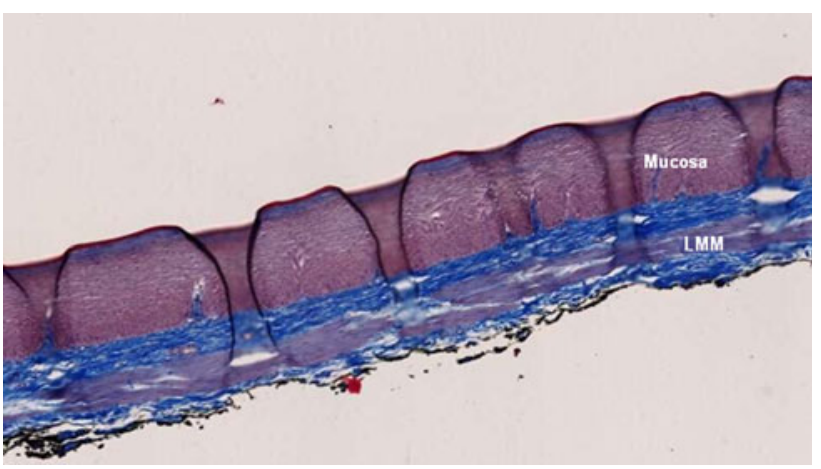

Fig. 4 Resected mucosa with lamina muscularis mucosae (Masson's trichrome stains). LMM lamina muscularis mucosae

39-48) mm (Fig. 4). The maximal length of EMR in the resected esophagus was calculated by measuring the length of absent lamina muscularis mucosae in the longitudinal sections of the esophagus (Fig. 5). An average length of 37.6 (range, 18-48) $\mathrm{mm}$ was found.

Before harvesting the esophagus, overinflation through the endoscope was used to reveal any zone of limited esophageal distension (= relative stricture) that corresponded to the midpoint of the circumferential EMR (Fig. 6A, B). To evaluate the degree of fibrosis, the percentage of fibrosis of the muscularis propria interna (MPI) and muscularis propria externa (MPE) were calculated in the two horizontal sections, respectively (Fig. 7). An average of $10 \%(0-25 \%)$ of fibrosis was found in the MPI layer and $7.2 \%(0-25 \%)$ in the MPE layer, respectively. There was no circumferential transmural fibrosis.

\section{Discussion}

Endoscopic mucosal resection was developed to treat early stage squamous cell carcinomas in the esophagus and dysplastic changes or early adenocarcinomas arising in Barrett's esophagus. This endoscopic intervention has become well accepted as a minimally invasive therapy. Our preclinical studies demonstrated the feasibility of long and circumferential EMRs in the sheep esophagus and showed an accurate depth of resection at the submucosal level $[2,18]$. A preliminary pilot study in humans with the rigid EMR esophagoscope gave excellent results for extensive noncircumferential resections of up to $12 \mathrm{~cm}^{2}$ [19]. In the present animal study, circumferential resection could be performed successfully in nine sheep without any intra- or postoperative complications.

It is known that the primary drawback of large circumferential resections is the development of cicatricial stenosis with transmural fibrosis at the midpoint of the resected area $[2,4]$. Chennat described the development of symptomatic 


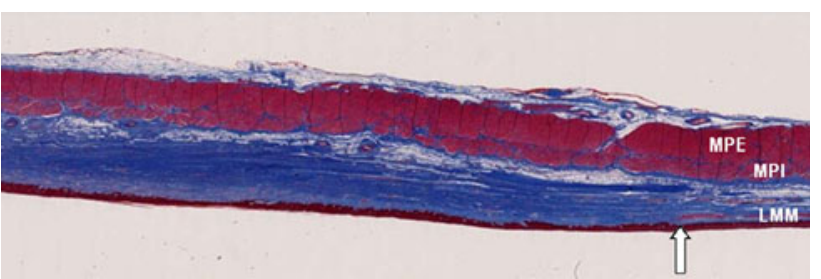

Fig. 5 Longitudinal section of the esophagus (Masson's trichrome stains). The arrow indicates the transition of resected area to normal esophagus with intact muscularis mucosae layer. MPE muscularis propria externa, MPI muscularis propria interna layer, $L M M$ lamina muscularis mucosae

stenosis in $37 \%$ of cases treated by circumferential EMR for complete Barrett's eradication [20].

In a previous animal experiment designed by our group [2], circumferential EMRs ranging from 2.2 to $5.5 \mathrm{~cm}$ in length were performed in 24 sheep. In $16(70 \%)$ animals, several dilatation sessions (mean 2; range 1-5) were necessary to prevent stricture formation during the postoperative follow-up. Only 8 of $24(30 \%)$ animals did not require any dilatation until full reepithelialization of the esophagus occurred. Histologically, transmural fibrosis was identified in 6 of $24(25 \%)$ animals. The remaining animals had scarring of the MPE of the esophageal wall.
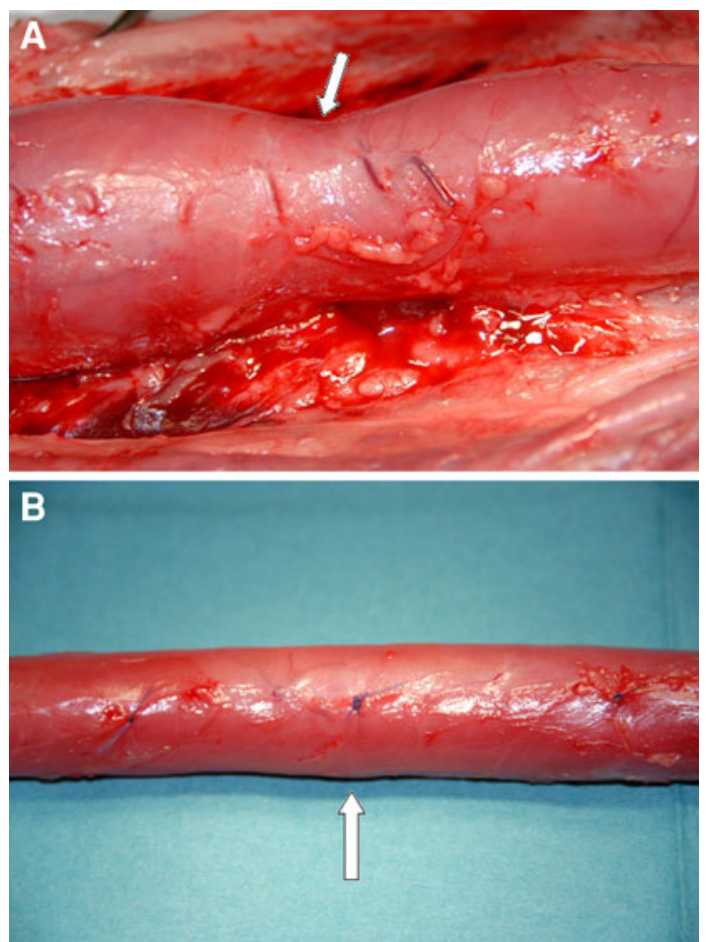

Fig. 6 A Macroscopic view of the overinflated esophagus during dissection. The arrow identifies the relative stricture, marked as the midpoint of the former circumferential EMR. B Macroscopic view of the dissected, stretched esophagus (arrow = maximal narrowing of the esophagus)

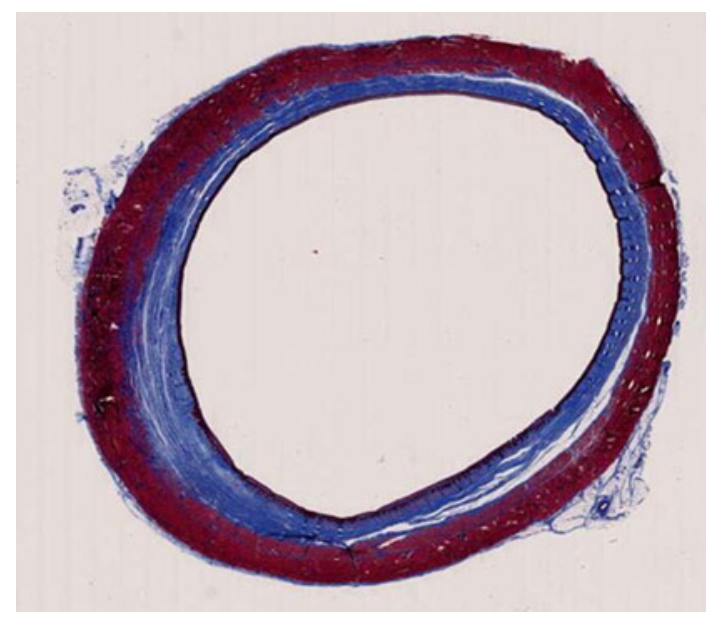

Fig. 7 Circumferential section of the esophagus with fibrotic changes in the submucosa and the MPI between 7 and 9 o'clock (Masson's trichrome stains)

In contrast, the present study displayed a completely different clinical and endoscopic picture. After injection of autologous keratinocytes, no stricture formation was observed and no dilatation was required. The clinical course was in general uneventful with no food impaction, and all animals were sacrificed as planned at the end of the 6-month follow-up.

This study provides preliminary findings that cutaneous keratinocytes, available from skin-tissue cultures, may promote early epithelialization when injected into the mucosal defect of EMRs and prevent deep wall ulceration, inflammation, and fibrosis resulting from prolonged bathing of the esophagus in saliva, food, and refluxate. The precise mechanism of the preventative effects of the implanted keratinocytes remains unclear. We are unable to ascertain whether the reepithelialized mucosal layer was created by the proliferation of injected keratinocytes because we did not label them. Preliminary studies that have attempted to label autologous keratinocytes with iron nanoparticles showed insufficient cell uptake of the iron. Tracing the fate of labeled keratinocytes for a period longer than 2 weeks in the ex vivo model was extremely difficult because of the proliferation of fibroblasts. In other studies [13], fluorescent dye has been used, but again, the fate of labeled keratinocytes could not be traced after passage of a long period of time for neither the in vivo nor ex vivo models. After a 6-month period, it is unlikely that labeled keratinocytes would still be present in the reepithelialized esophagus of our sheep model. The turnover of squamous cell epithelium is too fast in the esophagus to allow meaningful measurements over a long period of time. However, even if the mechanism of the reepithelialization process remains uncertain, whether the injected keratinocytes regenerate the mucosa or help the epithelial cells of the unresected area to migrate, its success is unquestionable. 
A split skin graft is easy to harvest in humans under local anesthesia and can be cultured by the technology of regenerative medicine, now commonly used for grafting of badly burned patients $[14,15]$. The denuded circumferential esophageal wound, which normally remains exposed to saliva, food, and refluxate for several weeks until complete healing occurs, undergoes a long-lasting inflammatory reaction that leads to scar formation and stricture. After injection of keratinocytes, the raw surface is supposed to undergo an accelerated reepithelialization process. In fact, only $10 \%$ of the circumferential MPI layer and $7.2 \%$ of the MPE layer in the midpoint of the EMRs showed fibrotic changes. By contrast, in a previous study by Pilloud et al. [2] transmural fibrosis was conspicuous in 6 of $24(25 \%)$ animals that underwent exactly the same procedure, albeit without keratinocyte injections.

Comparison of our results with other studies is difficult because of the differences in histological assessments. Most commonly, investigators have evaluated the success of a procedure only by the lack of transmural fibrosis or stricture formation and have not quantified the fibrotic changes at the different histological layers of the esophageal wall.

In our study, we quantified the degree of stricture by examining the percentage of fibrotic changes of the circumference at the midpoint level of the EMRs. The length of absent lamina muscularis mucosae was evaluated histologically after 6 months. Immediately after EMRs, the average length of the denuded esophagus was 7.3 (range, 6-9) cm, although 6-cm-long, circumferential EMRs were performed. This discrepancy in size was probably due to the natural tendency of the mucosa to undergo retraction after circumferential resection. The resected specimen of mucosa displayed the presence of the lamina muscularis mucosae over its entire length in 14 of 18 specimens $(77.7 \%)$.

After full reepithelialization of the esophagus, histological analysis showed an average length of absent lamina muscularis mucosae of 37.6 (range, 18-48) $\mathrm{mm}$. This shorter distance, compared with the resected specimen, was most likely due to cicatricial retraction. However, it also could be attributed to insufficient stretching of the esophagus before fixation in formalin, even though we always tried to reexpand the esophagus wound to the length of its original resection.

In our previous studies without prior injection of saline solutions for EMR, we observed one perforation in 86 animals $[2,18]$. In the present study, for preparation of the esophageal wall before EMR, a saline-adrenaline solution of $0.01 \%$ was injected into the submucosal layer to lift the epithelium and muscularis mucosae from the muscularis propria and thus reduce the risk of perforation and thermal injury. Adrenaline was added to reduce bleeding, which may compound the injection of autologous keratinocytes. In our series, no perforation or major bleeding occurred; therefore, the conditions for autologous keratinocyte injection were good.
Different techniques of EMR via flexible endoscopes have been reported: endoscopic multiband ligator [21], liftand-cut [22], cap-assisted [23], and/or free-hand techniques [24] with or without submucosal saline lift injection [20,25]. These techniques were used successfully to resect lesions of up to $2 \mathrm{~cm}$ in diameter but required several resections for larger lesions. With such techniques, the histological analysis of several patchwork specimens was challenging, and the correct staging of the lesion was problematic. Often, subsequent procedures were needed to complete the total resection, and long-term surveillance was required. The difficulty was complete eradication of the pathologic mucosa on the one hand and avoidance of resection-site overlaps with its inherent risk of perforation on the other. With the modified Storz rigid esophagoscope, two opposite resections can remove a circumferential mucosal surface of $24 \mathrm{~cm}^{2}$ during the same session within a short period of time (less than $30 \mathrm{~min}$ ). Histological assessment is easier and can stage neoplasia more accurately, because there are only two rectangular specimens for the entire surface area of $24 \mathrm{~cm}^{2}$. The disadvantage of this device is that the surgeon must be familiar with the rigid esophagoscope. Gastroenterologists who detect lesions in the esophagus are in general not used to working with rigid endoscopes and prefer techniques via the flexible endoscope. Further developments to implement our technique with a flexible endoscope are needed to permit more widespread use.

\section{Conclusions}

We have described a safe and effective treatment strategy for the management of high-grade dysplasia and early squamous or adenocarcinomas arising in the esophagus. In combination with the injection of autologous keratinocytes, prevention of strictures even in long circumferential resections could be achieved in the sheep model.

Disclosures Drs. Barbara F. Zuercher, Mercy George, Anette Escher, Elsa Piotet, Christos Ikonomidis, and Snezana Blant Andrejevic have no conflicts of interest or financial ties to disclose. The senior author Philippe Monnier will hold a financial relationship with the Storz Company, if the device reaches the market. For the present study, full intellectual independence of the authors was guaranteed.

\section{References}

1. Pellanda A, Grosjean P, Leoni S, Mihaescu A, Monnier P, Pasche P (1999) Abrasive esophageal cytology for the oncological follow-up of patients with head and neck cancer. Laryngoscope 109(10):1703-1708

2. Pilloud R, Jaquet Y, Monnier P (2005) Extensive circumferential endoscopic mucosal resection with a new rigid esophagoscope: an animal study. J Thorac Cardiovasc Surg 130(5):1399 
3. Jaquet Y, Pilloud R, Grosjean P, Radu A, Monnier P (2007) Extended endoscopic mucosal resection in the esophagus and hypopharynx: a new rigid device. Eur Arch Otorhinolaryngol 264(1):57-62

4. Mizuta H, Nishimori I, Kuratani Y, Higashidani Y, Kohsaki T, Onishi S (2009) Predictive factors for esophageal stenosis after endoscopic submucosal dissection for superficial esophageal cancer. Dis Esophagus 22(7):626-631

5. Yamashita T, Zeniya A, Ishii H, Tsuji T, Tsuda S, Nakane K, Komatsu M (2011) Endoscopic mucosal resection using a capfitted panendoscope and endoscopic submucosal dissection as optimal endoscopic procedures for superficial esophageal carcinoma. Surg Endosc 25(8):2541-2546

6. Eguchi T, Nakanishi Y, Shimoda T, Iwasaki M, Igaki H, Tachimori Y, Kato H, Yamaguchi H, Saito D, Umemura S (2006) Histopathological criteria for additional treatment after endoscopic mucosal resection for esophageal cancer: analysis of 464 surgically resected cases. Mod Pathol 19(3):475-480

7. Katada C, Muto M, Momma K, Arima M, Tajiri H, Kanamaru C, Ooyanagi H, Endo H, Michida T, Hasuike N, Oda I, Fujii T, Saito D (2007) Clinical outcome after endoscopic mucosal resection for esophageal squamous cell carcinoma invading the muscularis mucosae-a multicenter retrospective cohort study. Endoscopy 39(9):779-783

8. Tsunada S, Ogata S, Mannen K, Arima S, Sakata Y, Shiraishi R, Shimoda R, Ootani H, Yamaguchi K, Fujise T, Sakata H, Iwakiri R, Fujimoto K (2008) Case series of endoscopic balloon dilatation to treat a stricture caused by circumferential resection of the gastric antrum by endoscopic submucosal dissection. Gastrointest Endosc 67(6):979-983

9. Rajan E, Gostout C, Feitoza A, Herman L, Knipschield M, Burgart L, Chung S, Cotton P, Hawes R, Kalloo A, Kantsevoy S, Pasricha P (2005) Widespread endoscopic mucosal resection of the esophagus with strategies for stricture prevention: a preclinical study. Endoscopy 37(11):1111-1115

10. Mizutani T, Tadauchi A, Arinobe M, Narita Y, Kato R, Niwa Y, Ohmiya N, Itoh A, Hirooka Y, Honda H, Ueda M, Goto H (2010) Novel strategy for prevention of esophageal stricture after endoscopic surgery. Hepatogastroenterology 57(102-103):1150-1156

11. Nieponice A, Mcgrath K, Qureshi I, Beckman EJ, Luketich JD, Gilbert TW, Badylak SF (2009) An extracellular matrix scaffold for esophageal stricture prevention after circumferential EMR. Gastrointest Endosc 69:289-296

12. Honda M, Hori Y, Nakada A, Uji M, Nishizawa Y, Yamamoto K, Kobayashi T, Shimada H, Kida N, Sato T, Nakamura T (2011) Use of adipose tissue-derived stromal cells for prevention of esophageal stricture after circumferential EMR in a canine model. Gastrointest Endosc 73(4):777-784

13. Sakurai T, Miyazaki S, Miyata G, Satomi S, Hori Y (2007) Autologous buccal keratinocyte implantation for the prevention of stenosis after EMR of the esophagus. Gastrointest Endosc 66(1):167-173

14. Balasubramani M, Kumar TR, Babu M (2001) Skin substitutes: a review. Burns 27:534-544

15. Horch RE, Kopp J, Kneser U, Beier J, Bach AD (2005) Tissue engineering of cultured skin substitutes. J Cell Mol Med 9: 592-608

16. Liu JB, Miller LS, Goldberg BB, Feld RI, Alexander AA, Needleman L, Castell DO, Klenn PJ, Millward CL (1992) Transnasal US of the esophagus: preliminary morphologic and function studies. Radiology 184:721-727

17. Taniguchi DK, Martin RW, Trowers EA, Silverstein FE (1995) Simultaneous M-mode echoesophagram and manometry in the sheep esophagus. Gastrointest Endosc 41:582-586

18. Radu A, Grosjean P, Fontolliet C, Monnier P (2004) Endoscopic mucosal resection in the esophagus with a new rigid device: an animal study. Endoscopy 36(4):298-305

19. Monnier P, Jaquet Y, Radu A, Pilloud R, Grosjean P, Escher A, Piotet E, Blant SA (2010) Extensive ( 8 to $12 \mathrm{~cm}^{2}$ ) noncircumferential endoscopic mucosal resection for early esophageal cancer. Ann Thorac Surg 89(6):S2151-S2155

20. Chennat J, Konda VJ, Ross AS, de Tejada AH, Noffsinger A, Hart J, Lin S, Ferguson MK, Posner MC, Waxman I (2009) Complete Barrett's eradication endoscopic mucosal resection: an effective treatment modality for high-grade dysplasia and intramucosal carcinoma: an American single-center experience. Am J Gastroenterol 104(11):2684-2692

21. Fleischer DE, Wang GQ, Dawsey S, Tio TL, Newsome J, Kidwell J, Prifti S (1996) Tissue band ligation followed by snare resection (band and snare): a new technique for tissue acquisition in the esophagus. Gastrointest Endosc 44(1):68-72

22. Inoue $\mathrm{H}$, Takeshita $\mathrm{K}$, Hori $\mathrm{H}$, Muraoka $\mathrm{Y}$, Yoneshima $\mathrm{H}$, Endo M (1993) Endoscopic mucosal resection with a cap-fitted panendoscope for esophagus, stomach, and colon mucosal lesions. Gastrointest Endosc 39(1):58-62

23. Kamler JP, Borsatto R, Binmoeller KF (2002) Circumferential endoscopic mucosal resection in the swine esophagus assisted by a cap attachment. Gastrointest Endosc 55(7):923-928

24. Soehendra N, Binmoeller KF, Bohnacker S, Seitz U, Brand B, Thonke F, Gurakuqi G (1997) Endoscopic snare mucosectomy in the esophagus without any additional equipment: a simple technique for resection of flat early cancer. Endoscopy 29(5):380-383

25. Witteman BP, Foxwell TJ, Monsheimer S, Gelrud A, Eid GM, Nieponice A, O'Rourke RW, Hoppo T, Bouvy ND, Badylak SF, Jobe BA (2009) Transoral endoscopic inner layer esophagectomy: management of high-grade dysplasia and superficial cancer with organ preservation. J Gastrointest Surg 13(12):2104-2112 